\section{WIPE AWAY HIDDEN PERILS}

Most people have suffered at the hands of a computer virus, but it appears not everyone is aware of the real-life health risk their computer can pose. AF International, makers of computer and office cleaning products, warns that the fight against MRSA could be undermined by inadvertently allowing bacteria to flourish on touch-screens, telephones and other non-surgical equipment.

Its Phone-Clene wipes contain a strong bactericide and is ideal for use in office and administration areas. Phone-Clene is available in a convenient and portable 25 -wipe pack or a 100-wipe tub. The full AF range can be viewed at www.af-net.com.

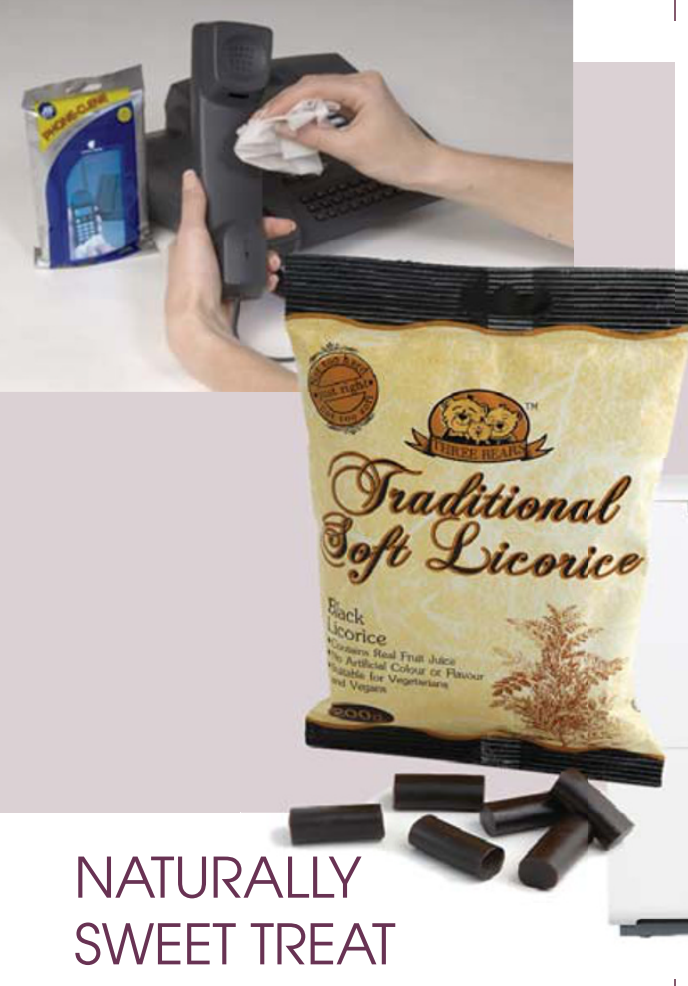

Three Bears Traditional Soft Licorice has just 23 calories in each piece. The traditional black licorice, which is suitable for vegetarians and vegans, contains real licorice extract, pineapple juice and natural colours and flavours - in fact, all the ingredients are natural, with no E numbers.

One of the main medicinal compounds in licorice root is a substance called glycyrrhizin (or glycyrrhic acid) which is 50 times sweeter than sugar. If you or your children have a sweet tooth, eating licorice may help keep your teeth healthy. According to Israeli scientists, licorice root could help reduce plaque, which causes tooth decay by reducing cavity-causing bacteria in the mouth. Three Bear Traditional Soft Licorice is available from health food stores or by mail order $(01782$ 825323 ) priced $\mathfrak{E} 1.69$ for $200 \mathrm{~g}$.

\section{INTERPROXIMAL PLAQUE REMOVAL}

Every dental professional knows the importance of effective plaque removal for preventing caries and periodontal diseases. New Colgate Total Interdental brushes have been developed with this in mind.

They have a unique triangular bristle shape in cross section and the Tri-Proxi brush has longer filaments which sit at a $120^{\circ}$ angle to the surrounding shorter filaments at the centre of the brush. This triangular shape adapts to the natural anatomy of interdental spaces, providing a better cleaning performance.

The unique handle configuration can be adapted to individual patient needs as extension improves access to posterior teeth also doubles as a cap for storage in between use for improved hygiene and portability.

For further information or to request a Colgate Total Interdental brush patient recommendation pad call the Colgate customer care team on 01483401901.

\section{STANDALONE DECONTAMINATION}

The Stanbridge Steri Combi is a complete standalone decontamination tower system for infection control of instrument washing and sterilising. For HTM 01-05 compliance, it will suit dental clinics and other healthcare practices where it will fit into a convenient corner to provide a central sterilising centre.

It has its own water treatment unit that provides clinical quality from piped tap water. The washer disinfector, with hot and cold fill, dries instruments at the end of the cycle. The instrument trays can then be moved directly to the steriliser, which has a vacuum unit for air removal, drying and pouching.

Call 01689 806500, email sales@stanbridge. co.uk or see www.stanbridge.co.uk for further information.

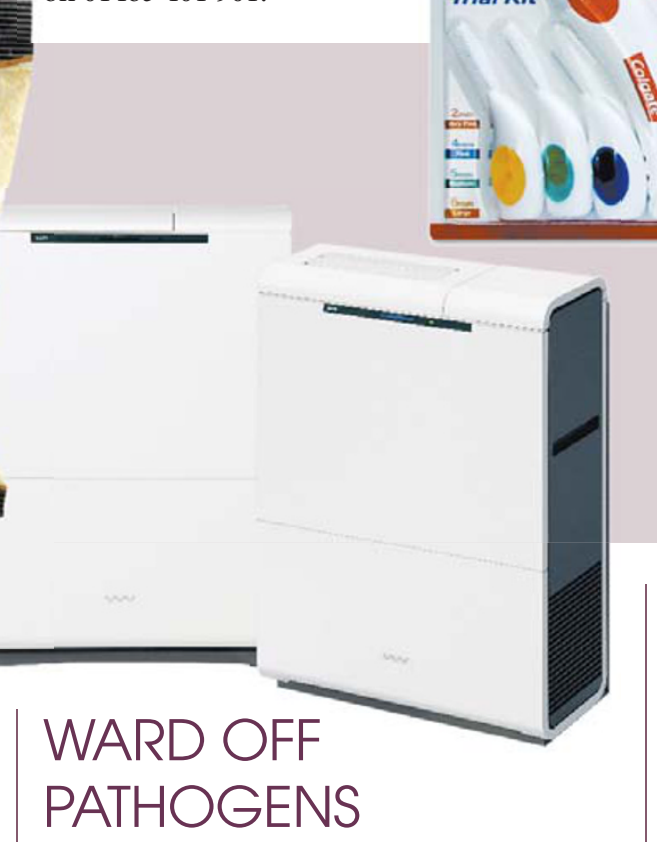

The new Sanyo virus washer protects healthcare staff from airborne pathogens by permanently inactivating airborne viruses and bacteria. The technology, which comes in the form of a moveable floor standing unit, is designed for use in sensitive and at risk areas such as surgeries and hospital waiting rooms, to protect staff and the general public from the spread of disease.

Electrolysed water runs over the air purification element in the virus washer and indoor air is then forced to circulate through the element, inactivating airborne viruses which pass through it. For further information contact Oceanair Distribution Ltd on 08456590022.

\section{THE NEED FOR}

\section{TEAMWORK}

Smile-on and Dental Protection (DPL) recently teamed up to communicate the need for teamwork. Visitors to the BDTA at ExCel were invited to the screening of the third episode in the Communications series brought about as a result of a collaboration by Smile-on and DPL.

The programme has been designed for the whole dental team and it can work effectively if watched and worked through together to stimulate discussion or can also be used individually.

Three hours of verifiable CPD is conferred on up to five participants, with CPD for additional viewers available. The programme licence allows it to be viewed and used by the whole dental team in practice or accessed on a home PC. For more information about the Communications series visit www.smile-on.com or call 0207400 8989. (Also see page 7.) 